\title{
Treatment of the elderly patient with acute myeloid leukaemia
}

\author{
BOB LÖWENBERG
}

Acute myeloid leukaemia (AML) is seen at all ages, but more than half of the patients with AML are 60 years of age or older (Brinker, 1982,1985). The incidence of AML at 60 years is approximately 4 per $100000 /$ year and at age 80 it is 15 per $100000 /$ year. Because of demographic changes and the projected increase of elderly people among the total population in the next decade, a significant increase in the incidence of cases of AML is foreseen (Liu Yin, 1993). Intensified cytotoxic therapy and bone marrow transplantation have improved the prognosis of AML in recent years in young and middle-aged adults. However, these modern approaches of therapy are of only little benefit to individuals of more than 60 years of age with AML. The causes of the differences of treatment outcome between middle-aged and elderly adults are probably multifactorial. Elderly people may suffer from AML which is a priori more resistant to chemotherapy. Furthermore, the abilities of aged individuals to tolerate the toxicity and morbidity brought along by intensive chemotherapy are evidently far more limited. Hence, older patients probably require treatment that is tailored to this age group. Only in recent years have specific efforts been made to develop therapy for the older age group. Here we shall briefly review the prognosis and outcome of current treatment in elderly patients with AML.

\section{REMISSION INDUCTION TREATMENT}

The initial cycles of chemotherapy always serve the objective of attaining a complete remission, a condition in which leukaemic cells can no longer be distinguished with cytomorphological or cytogenetic methods in the marrow, or at extramedullary sites, and in which blood cell counts recover to normal values. Remission induction chemotherapy usually involves a combination of the chemotherapeutic agents cytarabine and an anthracycline (daunomycin, idarubicin) or an anthracenedione (mitoxantrone). Following one or two cycles of chemotherapy a significant proportion of patients may enter complete remission. Should elderly patients with AML 
actually be submitted to remission induction chemotherapy, or would it be preferable to refrain and offer palliative support to these individuals? The prevailing opinion today is that intensive chemotherapy is the treatment of choice for elderly patients suffering from AML, if they are fit. A palliative wait-and-see strategy based mainly upon haematological and bacteriological supportive care has been directly compared with induction chemotherapy in a prospective randomized study in patients of over 65 years of age (Löwenberg et al, 1989). The purely supportive approach appeared a poor option and resulted not only in unfavourable survival but also in frequent hospitalization and a poor quality of life. The results of a variety of studies, all conducted in patients of 60 years of age or more, would indicate that these individuals have an approximately $50 \%$ probability of entering complete remission (Table 1). For comparison, as of today, among adults of less than 60 years, a proportion of $70 \%$ or more will attain complete remission following treatment with modern chemotherapy (Chapter 5). Thus, it is evident that the elderly population of patients respond significantly less favourably to induction treatment. The inverse relationship between age and complete remission rate is also apparent from a number of studies that enrolled patients covering a broad range of ages (Table 2). These results are indicative of a progressive decline in the response to chemotherapy with increasing age. Patients of 0-39 years, $40-59$ years, $60-84$ years or 70-84 years show average response rates of the order of $78,66,45$ and $35 \%$, respectively. How can we explain these differences? Are they due to differences of the dose or the choice of chemotherapeutic agents? Or are they perhaps due to the occurrence of AML of greater risk in patients aged 60 years and more? Only few prospective randomized studies have addressed the question of choice of drug and choice of dose and revealed differences in efficacy. In one study (Yates et al, 1982) daunomycin at a $30 \mathrm{mg} / \mathrm{m}^{2}$ dose (in combination with cytarabin) produced more complete responses $(47 \%)$ in patients over 60 years as compared to a daunomycin-cytarabin schedule with a $45 \mathrm{mg} / \mathrm{m}^{2}$

Table 1. Rates of complete response to induction chemotherapy in elderly patients with AML.

\begin{tabular}{lcccc}
\hline Reference & Patient number $(n)$ & Age & Chemotherapy & CR (\%) \\
\hline Keating et al (1981) & 59 & $60+$ & Rub/V & 49 \\
Kahn et al $(1984)^{\mathrm{b}}$ & 40 & $70+$ & DAT & 28 \\
Rees et al $(1986)^{\mathrm{b}}$ & 315 & $60+$ & DAT & 48 \\
Rees \& Gray (1987) & 176 & $60+$ & DAT & 43 \\
Löwenberg et al $^{\mathrm{b}}$ & 31 & $65+$ & DVA & 58 \\
Arlin et al $(1990)^{\mathrm{b}}$ & 99 & $60+$ & DA & 41 \\
& & & MA & \\
Liu Yin et al $(1991)^{\mathrm{a}}$ & 104 & $60+$ & MA & 58 \\
Mayer et al $(1994)^{\mathrm{b}}$ & 346 & $60+$ & DA & 47 \\
Dombret et al $(1995)^{\mathrm{a}}$ & 173 & $65+$ & DA & 59 \\
Stone et al $(1995)^{\mathrm{b}}$ & 388 & $60+$ & DA & 53 \\
\hline
\end{tabular}

${ }^{\mathrm{a}}$ Based upon prospective trials especially designed for patients of 60 years and older.

' Based upon prospective trials in adult patients with AML including elderly individuals.

$\mathrm{D}=$ daunomycin; $\mathrm{V}=$ vincristine; $\mathrm{M}=$ mitoxantrone; $\mathrm{CR}=$ complete remission; $\mathrm{A}=$ cytarabine;

$\mathrm{T}=$ thioguanine; $\mathrm{Rub}=$ rubidizone. 


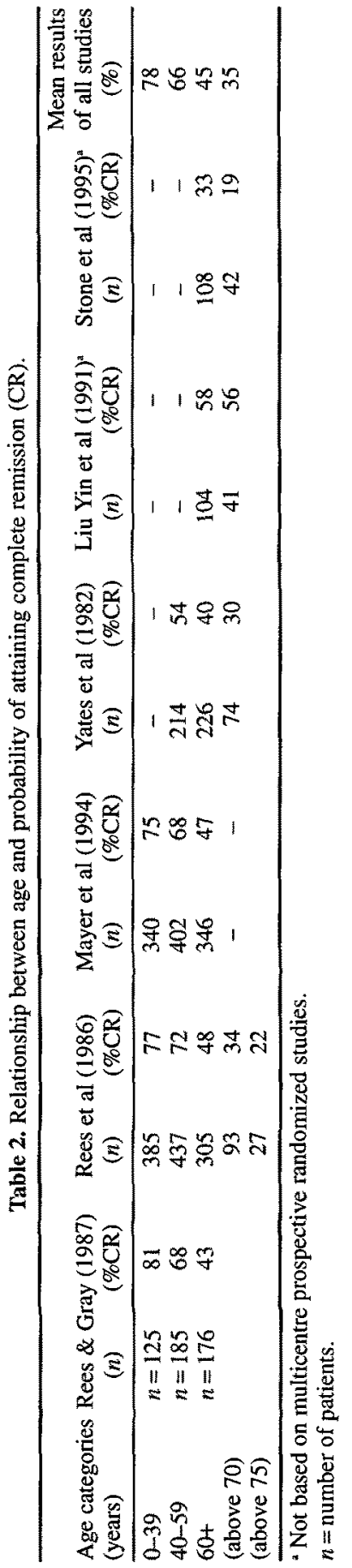


dose of daunomycin (31\%). The better response rate was due to a significantly reduced death rate in the daunomycin $30 \mathrm{mg}$ (dauno 30) group. Also, doxorubicin at $30 \mathrm{mg} / \mathrm{m}^{2}$ in combination with cytarabine gave comparatively worse results than did dauno 30 . However, in contrast, in patients less than 60 years a dauno 45 dose produced the best response results. This would indicate that optimal dose selection for elderly individuals might be different from that in younger adults. In another study (Kahn et al, 1984) a reduced dose of daunomycin $\left(50 \mathrm{mg} / \mathrm{m}^{2}\right.$ on day 1 as compared to $60 \mathrm{mg} / \mathrm{m}^{2}$ on days 1 through 3 ) did not result in better remission rates. In fact, the schedule with the attenuated dose schedule of daunomycin resulted in a reduced frequency of early and aplastic mortality. However, unfortunately, the lower dose schedule was also associated with a reduced antileukaemic response rate. Apparently, when chemotherapy is reduced too much in the interest of tolerability, the effectivity becomes inadequate. These observations, considered together, are clearly consistent with the notion that the balance between the benefits and risks of intensive chemotherapy is particularly delicate in older patients. Two studies addressed the question of the possible efficacy of mitoxantrone as compared to daunomycin, but they did not provide an unequivocal answer. In one study (Arlin et al, 1990) mitoxantrone permitted significantly better response rates in elderly patients. In contrast, in another study mitoxantrone and cytarabine as firstline therapy did not improve the likelihood of response (Liu Yin et al, 1991).

\section{COMPLICATIONS AND MORTALITY DURING REMISSION INDUCTION THERAPY}

Approximately $30 \%$ of patients who do not enter remission fail due to early death or death during the hypoplastic phase post-chemotherapy (Table 3). Death occurs during hospitalization in spite of supportive care, including antibiotics as well as red blood cell and platelet transfusions. A majority of the causes of death among these individuals are due to infections. The greater death rate would indicate that elderly individuals are less able to tolerate the consequences of severe infections. They succumb more frequently from the negative effects on cardiac, pulmonary or renal function or haemodynamic dysfunction. Indeed, there is experimental evidence in aged animals and elderly adults to suggest that haematopoietic regeneration in response to phlebotomy (Boggs and Patrene, 1985) or bacterial challenge (Williams et al, 1986) in aged recipients is impaired. The inability of aged subjects to regenerate haematopoiesis in response to cytotoxic therapy or other perturbations and to restore the haematopoietic equilibrium would predict that elderly patients with AML are less capable of responding to an increased haematopoietic demand and to compensate marrow suppression caused by cytotoxic therapy. There are also age-related changes that might affect the pharmacokinetics and pharmacodynamics of antineoplastic agents resulting in more intense or prolonged exposure (Balducci et al, 1989), e.g. as the result of hypoalbuminaemia, the bio- 
availability of protein-bound agents, and therefore also the toxicity, might increase. Hepatic metabolism and renal excretion may diminish at higher age, and these changes may alter the clearance or rate of inactivation of drugs. The reduced marrow regenerative abilities, the reduced tolerance of other organs to handle the toxic effects of drugs as well as the reduced elimination and metabolism of drugs might all explain why elderly patients are at increased risk of haematopoietic and other complications from intensive chemotherapy. The incidence of severe infections in elderly patients with AML on induction therapy is approximately 20\% (Stone et al, 1995). Approximately $10 \%$ of these patients die during or within 1 week following completion of chemotherapy (Liu Yin et al, 1991). If one considers the entire induction phase including the interval of postchemotherapy hypoplasia approximately $20 \%$ of patients expire (Yates et al, 1982; Rees and Gray, 1987; Arlin et al, 1990; Baudard et al, 1994). Thus, a substantial proportion of patients of over 60 years of age with AML do not survive remission induction therapy due to infectious or haemorrhagic complications during or following chemotherapy. Those who have experienced significant toxicity following the first cycle of chemotherapy are generally withdrawn prematurely from additional efforts of treatment. Thus, the obstacles to offering adequate therapy to older patients with AML are still quite formidable. There has been hope that the haematopoietic growth factors may have a role in hastening haematopoietic recovery, reducing the incidence and severity of complications and ultimately reducing mortality.

Table 3. Distribution of remission induction failures in elderly patients with AML.

\begin{tabular}{llcc}
\hline Reference & Age & $\begin{array}{c}\text { Resistant } \\
\text { leukaemia (\%) }\end{array}$ & $\begin{array}{c}\text { Early or } \\
\text { hypoplastic death }\end{array}$ \\
\hline Rees \& Gray (1987) & $60+$ & 15 & 32 \\
Yates et al (1982) & $60+^{\mathrm{b}}$ & 9 & 44 \\
Baudard et al (1984) & $60+$ & 45 & 12 \\
Stone et al (1995) & $60+$ & 22 & 25 \\
Dombret et al (1995) & $65+$ & 24 & 17 \\
" Death during chemotherapy & or following chemotherapy with no evidence of \\
persisting AML. \\
'Results of daunomycin (30 mg/m²)/cytarabine treatment arm. \\
n.r. not reported.
\end{tabular}

\section{CLINICAL USE OF HAEMATOPOIETIC GROWTH FACTORS TO MITIGATE HAEMATOPOIETIC TOXICITY}

Based on the assumption that GM-CSF or G-CSF might accelerate haematopoietic recovery, some studies have been conducted to explore a potential role for these agents. Early phase I-II studies with GM-CSF and G-CSF given after completion of chemotherapy in patients with AML indicated that there were no apparent negative effects of growth factor treatment on leukaemia clearance, leukaemic regrowth or recurrence of leukaemia (Bettelheim et al, 1991; Büchner et al, 1991; Ohno et al, 1994), 
even though in vitro data indicated that these factors would activate AML cell proliferation in culture (Löwenberg and Touw, 1993). Three randomized studies have dealt with the use of GM-CSF after chemotherapy (Löwenberg et al, submitted; Stone et al, 1995; Rowe et al, 1995). One other phase III study has explored a potential role of G-CSF (Dombret et al, 1995). In all four studies (see Table 4) an enhanced regeneration of neutrophils was apparent. A reduction of the length of neutropenia by 2-7 days was seen in G-CSF- and GM-CSF-treated patients. However, the reduction of the duration of neutropenia was not associated with a decrease of the death rate in any of these studies. In two studies, one study with GM-CSF (Rowe et al, 1995) and one study with G-CSF (Dombret et al, 1995) the complete response rate improved significantly to $60 \%$ and $70 \%$ respectively (Table 4 ). Why the complete response rate was better in only two of the four studies remains unexplained at the present time. In fact, the mortality rate was not reduced (Dombret et al, 1995; Löwenberg et al, submitted; Stone et al, 1995). Only one of the studies reported a reduction in the percentage of resistant leukaemia (Dombret et al, 1995) that would have contributed to a greater response probability in case of G-CSF therapy. In the latter study the time to attain complete remission was also shortened (Dombret et al, 1995). Notably, in none of the studies were the days of hospitalization of the patients or the incidence of bacterial or fungal infections modified. Survival had not changed in patients on either G-CSF or GM-CSF treatment, except in one study. In the latter study the placebotreated patients showed, for reasons which are not clear, a survival time significantly poorer than is usually seen in these patients (Rowe et al, 1995). Thus, the accumulating results of independent phase III studies do not convincingly establish the clinical usefulness of G-CSF or GM-CSF after chemotherapy. Apparently, the haematopoietic effect of haematopoietic growth factors in vivo is too limited to be exploited in the clinical setting. For the time being therefore the general or prophylactic use of these biosynthetic drugs in the standard treatment of elderly patients with AML cannot be recommended. In 1994, a new growth factor, thrombopoietin, has been cloned. This factor has now entered clinical trial. It will be of interest to investigate whether the clinical use of this factor in older patients with AML will be able to reduce the severity or length of duration of thrombocytopenia and also make treatment in these individuals less cumbersome by reducing the requirements for platelet transfusion and decreasing the problems of platelet support in allo-immunized patients.

\section{RESISTANCE TO THERAPY}

Resistance of AML to chemotherapy has been a major cause of treatment failure in patients of over 60 years of age with AML. In a number of recent studies the proportion of patients showing primary resistance to chemotherapy as the cause of remission induction failure was estimated at $15 \%$ or more (Table 3). Why would AML in elderly individuals be relatively unresponsive to chemotherapy? Are the intrinsic unfavourable prognostic 


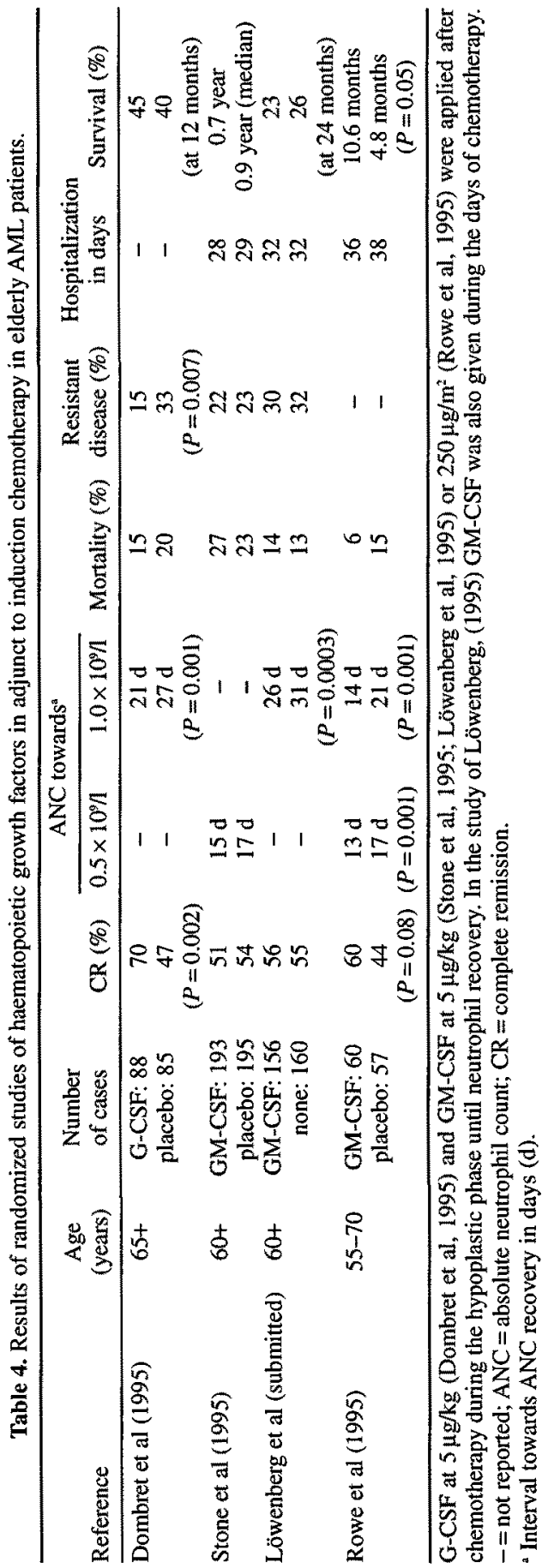


features of AML more frequently expressed in elderly patients? The answer to these questions is at the present time based mainly on circumstantial evidence rather than on a solid set of experimental data. For instance, M0 FAB cytological subtype (Stasi et al, 1994), unfavourable cytogenetic abnormalities (e.g. chromosome 5 and 7 abnormalities) (see Chapter 1), MDR-1 expression (Arceci, 1993; Lamy et al, 1994) (see Chapter 10), bcl-2 positivity (Campos et al, 1993), CD34 positivity (Geller et al, 1990; Guinot et al, 1991; Solary et al, 1992; Boekhorst et al, 1993a), high levels of autonomous proliferation in culture (Löwenberg et al, 1993; Boekhorst et al, 1993b) are all highly adverse predictors of treatment outcome. There are insufficient data available to indicate that the incidence of these poor risk subtypes is increased among elderly patients with AML. The results of some retrospective studies would suggest that elderly patients generally present more often with secondary AML following myeloproliferative disorders and myelodysplasia (13\% versus $4 \%$ ) (Baudard et al, 1994). Favourable subtypes of AML (e.g. AML M3 1\% versus 12\%) and subtypes with unfavourable cytogenetic abnormalities are comparatively underrepresented among patients of over 60 years of age (Baudard et al, 1994; Dastugue et al, 1995). This would a priori set an unfavourable stage for treatment outcome. AMLs evolving from a myelodysplasia as well as AMLs secondary to chemotherapy applied previously for another medical condition are generally poor-risk conditions. The myelodysplastic syndromes are more often seen in elderly patients and are likely to contribute to the increased incidence of high-risk AML in older subjects. AMLs developing after progression from MDS generally respond poorly to chemotherapy, thus providing another explanation for the unsatisfactory response to treatment (Keating et al, 1981; Liu Yin, 1993).

\section{CLINICAL USE OF HAEMATOPOIETIC GROWTH FACTORS TO ENHANCE CHEMOSENSITIVITY}

The probability that elderly individuals with newly diagnosed AML will attain a complete remission is approximately $50 \%$. Of those who enter remission, the majority lose the remission status within 2 years, and only few become long-term survivors. Thus, recurrent disease poses a significant problem and is a main determinant of unfavourable outcome. Of those who do not at all enter a complete remission, a significant number appear primarily refractory to chemotherapy. Both because of the high relapse probability and the high rate of primary treatment resistance attempts are being made to overcome resistance (Arceci, 1993). The subject of drug resistance is extensively discussed in Chapter 10 . The haematopoietic growth factors have also received considerable interest because of their ability in vitro to enhance the killing of leukaemic blasts or leukaemic colony-forming cells by cytotoxic drugs. Exposure of leukaemic cells to cytarabine in the context of growth factor stimulation has shown an increase in formation of Ara-CTP, increased DNA uptake of radiolabelled cytarabine in leukaemic cells and, in addition, enhancement of cytotoxicity 
(Bhalla et al, 1988; Cannistra et al, 1989; Miyauchi et al, 1989; Santini et al, 1990; Boekhorst et al, 1992; Hiddemann et al, 1992; Boekhorst et al, 1994). These in vitro observations have laid the basis for introducing haematopoietic growth factors in clinical protocols. The simultaneous administration of haematopoietic growth factors in vivo with chemotherapy would prime the leukaemic cells and render the cells more susceptible to killing by cytarabine. At the present time, clinical studies addressing the question of enhancement of chemotherapy effectivity by haematopoietic growth factors are scarce. The results of a randomized phase II study in patients with myelodysplastic syndromes of median age of 59 years submitted to daunomycin/cytarabine chemotherapy and G-CSF during chemotherapy, as well as post-chemotherapy, were indicative of a trend of better CR rates, but the difference was not significant (Ossenkoppele et al, submitted). In a study in elderly patients with AML of 60 years and over, GM-CSF was applied during and after chemotherapy, but no differences in CR rate, relapse rate or survival were apparent in the case of GM-CSF therapy (Löwenberg et al, submitted). Thus, for the time being there is no evidence to support a role for growth factor priming of patients with AML, although the data are still scarce. One might wonder why an effect of cytotoxicity enhancement that has been so apparent in vitro would not be seen in vivo. One explanation for the in vitro-in vivo discrepancy of growth factor priming of chemotherapy is that the in vitro colony-forming cells are not truly representative of the in vivo leukaemic re-populating progenitors, and therefore the in vitro data would not predict for in vivo efficacy. Another explanation is that the dose of growth factor applied in vivo (usually $5 \mu \mathrm{g} / \mathrm{kg}$ ) is inadequate to stimulate AML progenitors fully. In fact it has become clear that much higher dosages of haematopoietic growth factors would be needed for optimal stimulation and mobilization of normal stem cells in vivo, and, by analogy, one might hypothesize that leukaemic stem cells would likewise require significantly greater concentrations of growth factor stimulation. Additional studies are clearly needed to clarify a potential role of the administration of HGFs for modifying cytotoxicity of chemotherapeutic agents in AML.

\section{OUTCOME OF TREATMENT AND PROGNOSIS}

Patients aged 60 years once induced into remission are usually consolidated with additional cycles of chemotherapy and rarely with bone marrow transplantation or high-dose chemotherapy. Bone marrow transplants are currently being carried out in patients of more than 50 years with satisfactory results, but only infrequently in patients aged 60 years and above (Cahn et al, 1995). A randomized study of post-remission therapy with three dose levels of cytarabine $\left(100 \mathrm{mg} / \mathrm{m}^{2}, 400 \mathrm{mg} / \mathrm{m}^{2}\right.$ and $\left.3 \mathrm{~g} / \mathrm{m}^{2}\right)$ showed a clear dose-effect relationship in adults less than 60 years of age, but this relationship was not apparent in older subjects. The schedule of cytarabine at $3 \mathrm{~g} / \mathrm{m}^{2}$ resulted in significantly reduced relapse probability and superior survival in patients aged 60 years and below (Mayer et al, 1994). However, 
the advantage of dose escalation of cytarabine was not apparent in older individuals. Therefore, high-dose chemotherapy may not be beneficial to the elderly with AML. Of patients of 60 years and older treated with chemotherapy, survival at 2 years has been estimated at approximately $20 \%$ (Rees et al, 1986; Löwenberg et al, 1989) and at 4-5 years at $10 \%$ or less (Rees et al, 1986; Liu Yin et al, 1991; Baudard et al, 1994). Median diseasefree survival is approximately 10 months (Liu Yin et al, 1991; Baudard et al, 1994) and approximately $30 \%$ of patients survive free of leukaemia at 2 years (Rees et al, 1986; Baudard et al, 1994). Disease-free survival at 4-5 years is of the order of 5-14\% (Rees et al, 1986; Liu Yin et al, 1991; Baudard et al, 1994; Mayer et al, 1994). Thus, generally only a minor fraction of elderly patients submitted to intensive chemotherapy at the end become long-term survivors (Table 5). These results, while being meagre, yet represent an underestimate of disease outcome in elderly individuals with AML. Many patients are not referred to the hospital at all for chemotherapy, elect themselves not to be treated, or are considered ineligible to receive chemotherapy for clinical reasons. If one takes these patients into account, the overall prognosis of elderly patients with AML is far more dismal than the results of prospective studies would indicate. One report estimated that only approximately one-third of elderly patients were selected to enter treatment protocols (Baudard et al, 1994). In fact, the variability of patient selection leading to the inclusion of patients with variable risks into individual studies might help to explain the differing outcomes of treatment in study reports. In one study of more than 400 cases, an additional number of 196 patients were identified as not having entered into the trial (Yates et al, 1982). The single most important reason for exclusion of patients from trial therapy was related to the high age and poor clinical state of the patients. The median age of non-trial patients (67 years) was significantly greater than the age (53 years) of the trial group. Based on these considerations one might assume that the 4 year survival of elderly patients will be $5 \%$ or less. Therefore, the decision to treat or not to treat is an important choice in these individuals. It makes sense to select patients for induction treatment only if they are in reasonably good clinical condition. Further, one could argue that it makes sense to proceed with additional cycles of therapy only if they tolerate the first cycle of treatment with no major complications and if a haematological response to the first

Table 5. Outcome of chemotherapy in elderly patients with AML.

\begin{tabular}{|c|c|c|c|c|}
\hline \multicolumn{2}{|c|}{ Patients } & $\begin{array}{l}\text { Disease free } \\
\text { survival }(\%)\end{array}$ & $\begin{array}{c}\text { Overall } \\
\text { survival }(\%)\end{array}$ & Reference \\
\hline 104 & $60+$ & $15^{\mathrm{a}}$ & $10^{a}$ & Liu Yin et al (1991) \\
\hline 108 & $60+$ & $6^{\mathrm{b}}$ & $4^{b}$ & Baudard et al (1994) \\
\hline 315 & $60+$ & $5^{b}$ & $10^{b}$ & Rees et al (1986) \\
\hline 346 & $60+$ & $14^{\mathrm{c}}$ & n.r. & Mayer et al (1994) \\
\hline
\end{tabular}


therapy cycle is evident. This practical approach may serve to avoid 'overtreatment' in elderly patients, i.e. with no benefit and at the expense of prolonged hospitalization.

\section{SUMMARY}

Individuals of 60 years living in western countries generally have a mean life expectancy of 20 years at least. Therefore, when aged individuals present with AML, it is a necessity and a challenge to treat them as efficiently as possible. AML is mainly a disease of the elderly and accounts for more than $50 \%$ of its incidence among the general population. The treatment of older individuals with AML has remained difficult and its success is still limited. While in adults with AML of less than 60 years complete responses above $65 \%$ and survival rates of $35 \%$ are commonly obtained, progress in the treatment of elderly patients has been relatively small. As of today, approximately $50 \%$ of older patients may be induced into remission with chemotherapy, and, among these complete responders, only approximately 1 in 10 will survive free of leukaemia beyond 4 years after diagnosis. In fact, on one hand, these results represent the rationale and motivation for offering chemotherapy to the older population. On the other hand, they emphasize that major obstacles to better cure rates still exist. These stumbling blocks apparently relate to the restricted tolerance of older subjects to the exposure of chemotherapy and probably also a greater probability of unresponsiveness of the leukaemia to cytotoxic therapy. The haematopoietic growth factors still hold some promise and may improve outcome, but for the time being there is insufficient direct evidence to indicate a defined and established role. It is evident that new avenues should be pursued and trials specifically designed for elderly people with AML be conducted. These trials would need to address questions related to the choice of chemotherapeutic drugs (e.g. idarubicin versus mitoxantrone), their dose and schedule selection, the use of multidrug resistance modulators (to overcome intrinsic drug non-responsiveness), and the optimal clinical use of haematopoietic growth factors, including thrombopoietin. Since trials addressing specific questions regarding the development of treatment of elderly patients with AML have remained scarce, the initiation of these studies is sorely needed. One may hope that these clinical trials will provide some of the necessary answers and new clues, and will be useful to advance future therapy of elderly AML patients.

\section{REFERENCES}

Arceci RJ (1993) Clinical significance of P-glycoprotein in multidrug resistance malignancies. Blood 81: 2215-2222.

Arlin Z, Case DC, Moore J et al (1990) Randomised multicenter trial of cytosine arabinoside with mitoxantrone or daunorubicin in previously untreated adult patients with acute nonlymphocytic leukemia (ANLL). Leukemia 4: 177-183. 
Balducci L, Parker M, Sexton W \& Tantranond P (1989) Pharmacology of antineoplastic agents in the elderly patient. Seminars in Oncology 16: 76-84.

Baudard M, Marie JP, Cadiou M et al (1994) Acute myelogenous leukemia in the elderly: retrospective study of 235 consecutive patients. British Journal of Haematology 86: 82-91.

Bettelheim P, Valent $\mathrm{P}$, Andreeff $\mathrm{M}$ et al (1991) Recombinant human granulocyte-macrophage colonystimulating factor in combination with standard induction chemotherapy in de novo acute myeloid leukemia. Blood 77: 700-711.

Bhalla K, Birkhofer M, Arlin Z et al (1988) Effect of recombinant GM-CSF on the metabolism of cytosine arabinoside in normal and leukemic human bone marrow cells. Leukenia 2: 810-813.

Boekhorst PAW te, Löwenberg B \& Sonneveld P (1992) Stimulation by granulocyte colony stimulating factor (G-CSF), interleukin-3 (IL-3) or granulocyte-macrophage colony stimulating factor (GM-CSF) enhances chemosensitivity by clonogenic acute myeloid leukemia cells. Blood 80: 432.

Boekhorst PAW te, Leeuw K de, Schoester M et al (1993a) Predominance of functional multidrug resistance (MDR-1) phenotype in CD34+ acute myeloid leukemia cells. Blood 82: 3157-3162.

Boekhorst PAW te, Sonneveld P \& Löwenberg B (1993b) Autonomous growth and drug resistance mechanisms in adult acute myeloid leukemia. Experimental Hematology 21: 250.

Boekhorst PAW te, Löwenberg B \& Sonneveld P (1994) Hematopoietic growth factor stimulation and cytarabine cytotoxicity in vitro: effects in untreated and relapsed or primary refractory acute myeloid leukemia. Leukemia 8: 1480-1486.

Boggs DR \& Patrene KD (1985) Hematopoiesis and aging III: Anemia and a blunted erythropoietic response to hemorrhage in aged mice. American Journal of Hematology 19: 327-338.

Brinker H (1982) Population-based age and sex specific incidence rates in the 4 main types of leukemia. Scandinavian Journal of Haematology 29: 241-249.

Brinker H (1985) Estimate of overall treatment results in acute non-lymphocytic leukemia based on age specific rates of incidence and complete remission. Cancer Treatment Reports 69: 5-11.

Büchner Th, Hiddemann W, Koenigsmann $M$ et al (1991) Recombinant human granulocytemacrophage colony-stimulating factor after chemotherapy in patients with acute myeloid leukemia at higher age or after relapse. Blood 78: 1190-1197.

Cahn JY, Labopin M, Mandelli F et al (1995) Autologous bone marrow transplantation for first remission acute myeloblastic leukemia in patients older than 50 years: a retrospective analysis of the European Bone Marrow Transplant Group, Blood 85: 575-579.

Campos L, Rouault JP, Sabido O et al (1993) High expression of bcl-2 protein in acute myeloid leukemia cells is associated with poor response to chemotherapy. Blood 81: 3091-3096.

Cannistra SA, Groshek P \& Griffin JD (1989) Granulocyte-macrophage colony-stimulating factor enhances the cytotoxic effects of cytosine arabinoside in acute myeloblastic leukemia and in the myeloid blast crisis phase of chronic myeloid leukemia. Leukemia 3: 328-334.

Dastugue N, Payen C, Lafage-Pochitaloff M et al (1995) Prognostic significance of karyotype in de novo adult acute myeloid leukemia. Leukemia 9: 1491-1498.

Dombret H, Chastang C, Fenaux P et al (1995) A controlled study of recombinant human granulocyte colony-stimulating factor in elderly patients after treatment for acute myelogenous leukemia. New England Journal of Medicine 332: 1678-1683.

Geller RB, Zahurak M, Hurwitz CA et al (1990) Prognostic importance of immunophenotyping in adults with acute myelocytic leukaemia: the significance of the stem-cell glycoprotein CD34 (My 10). British Journal of Haematology 76: 340-347.

Guinot M, Sanz GF, Sempere A et al (1991) Prognostic value of CD34 expression in de novo acute myeloblastic leukemia. British Journal of Haematology 15: 533-534.

Hiddemann W, Kiehl M, Zühlsdorf M et al (1992) Granulocyte-macrophage colony-stimulating factor and interleukin-3 enhance the incorporation of cytosine arabinoside into the DNA of leukemic blasts and the cytotoxic effect of clonogenic cells from patients with acute myeloid leukemia. Seminars in Oncology 19: 31-37.

Kahn SB, Begg CB, Mazza JJ et al (1984) Full dose versus attenuated dose daunomycin, cytosine arabinoside and 6-thio-guanine in the treatment of acute nonlymphocytic leukemia in the elderly. Journal of Clinical Oncology 2: 865-870.

Keating MJ, Mc. Credie KB, Benjamin RS et al (1981) Tteatment of patients over 50 years of age with acute myelogenous leukemia with a combination of rubidizone and cytosine arabinoside, vincristine and prednisone (ROAP), Blood 58: 584-590.

Lamy T, Goasguen JE, Mordelet E et al (1994) P-Glycoprotein (P-170) and CD34 Expression in adult acute myeloid lenkemia (AML). Leukemia 11: 1879-1883. 
Liu Yin JA (1993) Acute myeloid leukaemia in the elderly: biology and treatment. British Journal of Haematology 83: 1-6.

Liu Yin JA, Johnson PRE, Davies JM et al (1991) Mitozantrone and cytosine arabinoside as first-line therapy in elderly patients with acute myeloid leukemia. British Journal of Haematology 79: $415-420$.

Löwenberg B, Zittoun R, Kerkhofs $\mathrm{H}$ et al (1989) On the value of intensive remission-induction chemotherapy in elderly patients of $65+$ years with acute myeloid leukemia: a randomized phase III study of the EORTC Leukemia Group. Journal of Clinical Oncology 1: 1268-1274.

Löwenberg B \& Touw IP (1993) Hematopoietic growth factors and their receptors in acute leukemia. Blood 81: 281-292.

Löwenberg B, Putten WLJ van, Touw IP et al (1993) Autonomous proliferation of leukemic cells in vitro as a determinant of prognosis in adult acute myeloid leukemia. New England Journal of Medicine 328: 614-619.

Löwenberg B, Suciu S, Zittoun R et al (1995) GM-CSF during as well as after induction chemotherapy (CT) in elderly patients with acute myeloid leukemia (AML). The EORTC-HOVON phase III trial (AML 11). Blood 86 (supplement 10): 43 (abstract).

Mayer RJ, Davis RB, Schiffer CA et al (1994) Intensive postremission chemotherapy in adults with acute myeloid leukemia. New England Journal of Medicine 331: 896-903.

Miyauchi J, Kelleher CA, Wang CH et al (1989) Growth factors influence the sensitivity of leukemic stem cells to cytosine arabinoside in culture. Blood 73: 1272-1278.

Ohno R, Naoe T, Kanamaru A et al (1994) A double-blind controlled study of granulocyte colonystimulating factor started two days before induction chemotherapy in refractory acute myeloid leukemia. Blood 83: 2086-2092.

Ossenkoppele GJ, Verhoef GEG, Bolt B v.d. et al (1995) Randomized phase II study on the value of G-CSF (filgrastim) in combination with standard induction chemotherapy in myelodysplastic syndromes (MDS). Blood 86 (supplement 1): p 338a (abstract).

Rees JKH, Gray RG, Swirsky D \& Hayhoe FGJ (1986) Principal results of the medical research council's 8th acute myeloid leukemia trial. Lancet ii: 1236-1241.

Rees JKH \& Gray R (1987) Comparison of $1+5$ DAT and 3 + 10 DAT followed by COAP or MAZE consolidation therapy in the treatment of acute myeloid leukemia: MRC ninth AML trial. Seminars in Oncology 14: 32-36.

Rowe JM, Andersen JW, Mazza JJ et al (1995) A randomized placebo-controlled phase III study of granulocyte-macrophage colony-stimulating factor in adult patients ( $>55$ to 70 years of age) with acute myelogenous leukemia: a study of the Eastern Cooperative Oncology Group (E 1490). Blood 86: 457-462.

Santini V, Nooter K, Delwel R \& Löwenberg B (1990) Susceptibility of acute myeloid leukemia (AML) cells from clinically resistant and sensitive patients to daunomycin (DNR): assessment in vitro after stimulation with colony stimulating factors (CSFs). Leukemia Research 14: $377-380$.

Solary E, Casanovas RE, Campos L et al (1992) Surface markers in adult acute myeloblastic leukemia: correlation of CD19+, CD34+ and CD14+/DR- phenotypes with shorter survival. Leukemia 6: 393-399.

Stasi R, Poeta GD, Venditti A et al (1994) Analysis of treatment failure in patients with minimally differentiated acute myeloid leukemia (AML-M0). Blood 83: 1619-1625.

Stone RM, Berg DT, George SL et al (1995) Granulocyte-macrophage colony-stimulating factor after initial chemotherapy for elderly patients with primary acute myelogenous leukemia. New England Journal of Medicine 332: 1671-1677.

Williams LH, Udupa KB \& Lipschitz DA (1986) Evaluation of the effect of hematopoiesis in C57BL/6 mouse. Experimental Hematology 14: 827-832.

Yates J, Glidewell O, Wiernik P et al (1982) Cytosine arabinoside with daunomycin or adriamycin for therapy of acute myelocytic leukemia: a CALGB study. Blood 60: 454-463. 